\section{Central retinal vein occlusion: modifying current treatment protocols}

M Ashraf ${ }^{1}$, AAR Souka ${ }^{1}$ and RP Singh ${ }^{2}$

\begin{abstract}
Central retinal vein occlusion (CRVO) is a common retinal vascular disorder that can result in severe visual acuity loss. The randomized control study, CRUISE, helped establish anti-VEGFs as the standard of care in cases with CRVO. The extension studies for CRUISE; HORIZON and RETAIN showed that not all visual gains are maintained beyond the first year. In addition, patients showed different behavior patterns; with some patients showing complete response with few recurrences, whereas others showed partial or even no response with multiple recurrences. Long-term follow-up demonstrated that patients responding poorly to anti-VEGFs tended to do so early in the course of treatment. It also demonstrated the effectiveness of a pro re nata (PRN) protocol for improving vision and maintaining these gains over long-term follow-ups. The SHORE study further illustrated this point by demonstrating that there were minimal differences in visual outcomes between patients receiving monthly injections and patients being treated PRN. In this review we analyzed the data from the major randomized clinical trials (RCT) that looked at anti-VEGFs as the primary treatment modality in patients with CRVO (CRUISE and the extension studies HORIZON and RETAIN for ranibizumab as well as GALILEO and COPERNICUS for aflibercept). In addition, we looked at SCORE and GENEVA to help determine whether there is a place for steroids as a first line therapy in current treatment practice. We then explored alternative treatment regimens such as laser therapy and switching between anti-VEGF agents and/or steroids for non or partially responding patients. Finally, we propose a simplified modified treatment algorithm for patients with CRVO for better long-term outcomes in all types of responders.
\end{abstract}

Eye (2016) 30, 505-514; doi:10.1038/eye.2016.10; published online 12 February 2016

\section{Introduction}

Central retinal vein occlusion (CRVO) is an acute retinal vascular condition that can severely affect visual acuity. ${ }^{1}$ Previous studies estimated that $\sim 2.5$ million people worldwide are affected by CRVO and about 13.9 million people are affected by branch retinal vein occlusion (BRVO). ${ }^{2}$ Visual loss after CRVO commonly occurs as a result of macular edema, macular ischemia, or in more advanced stages, vitreous hemorrhage, and neovascularization. ${ }^{3}$

CRVOs have been traditionally classified into ischemic and non-ischemic based on the degree of capillary non-perfusion on fluorescein angiography. ${ }^{4}$ The most commonly used criteria for ischemic CRVO was determined by the CVOS study group, which defined ischemic CRVO as at least 10 disc areas of capillary non-perfusion. ${ }^{5}$

Differentiating both subtypes is important because it allows us to predict the natural history for these patients and how they will respond to therapy. The ischemic subtype of CRVO accounts for about $20 \%$ of cases and is associated with worse initial presenting visual acuity (VA) and poor visual prognosis even after edema resolution. ${ }^{6}$ A cohort study showed that presenting VA in ischemic CRVO patients was $(6 / 30)$ or better in only $1 \%$ of ischemic CRVO patients compared with $78 \%$ of non-ischemic patients. ${ }^{6}$ Furthermore, final VA after resolution was better than $6 / 30$ in only $12 \%$ of ischemic CRVO patients compared with $83 \%$ in non-ischemic patients. Stratifying patients early based on the perfusion state and the initial presenting visual acuity is useful in predicting outcomes. ${ }^{6,7}$

\author{
${ }^{1}$ Ophthalmology \\ Department, Faculty of \\ Medicine, Alexandria \\ University, Alexandria, \\ Egypt \\ ${ }^{2}$ Cole Eye Institute, \\ Cleveland Clinic, \\ Cleveland, OH, USA \\ Correspondence: \\ M Ashraf, Ophthalmology \\ Department, Faculty of \\ Medicine, Alexandria \\ University, In front of 27 \\ Maarouf Rasafi Street, Kafr \\ Abdou, Roshdi, Alexandria, \\ Egypt \\ Tel/Fax: +20 1278647125. \\ E-mail: Moah384@gmail.com \\ Received: 22 September \\ 2015 \\ Accepted in revised form: \\ 8 December 2015 \\ Published online: \\ 12 February 2016
}




\section{Current treatment modalities}

\section{Anti-vascular endothelial growth factors (anti-VEGF) background}

Anti-VEGFs have become the standard of care for treating CRVO. There are three major anti-VEGF medications that are currently used for the treatment of macular edema associated with CRVO. Ranibizumab (Lucentis, Genetech, Inc., South San Francisco, CA, USA) is a $48-\mathrm{kDa}$ recombinant humanized immunoglobulin-G1 kappa isotype antibody fragment that binds all isoforms of VEGF-A. ${ }^{8}$ Bevacizumab (Avastin, Genentech, Inc.) is a 149-kDa full-length humanized monoclonal immunoglobulin-G1 antibody that binds all isoforms of VEGF-A. ${ }^{9}$ Aflibercept (Eylea, Regeneron Pharmaceuticals, Inc., Tarrytown, NY, USA), previously known as VEGF TrapEye, is a $115-\mathrm{kDa}$ recombinant fusion protein consisting of VEGF extracellular binding domains. In addition to its competitive inhibition of VEGF, aflibercept also binds placental growth factors 1 and 2. Aflibercept and ranibizumab are currently approved by the Food and Drug Administration (FDA) and has received marketing authorization from the European Medicines Agency (EMA) for macular edema (MO) associated with CRVO, whereas bevacizumab is unlicensed for intraocular injections. ${ }^{10}$

\section{What we learned from the major clinical trials}

\section{Ranibizumab}

The first major randomized clinical trial (RCT) looking into the effects of anti-VEGFs (and in particular ranibizumab) was the CRUISE study. ${ }^{11}$ Table 1 summarizes the major ranibizumab CRVO trial CRUISE and its extension studies HORIZON and RETAIN. In brief patients were randomized into three groups; $0.3 \mathrm{mg}$ ranibizumab, $0.5 \mathrm{mg}$ ranibizumab, and sham $/ 0.5 \mathrm{mg}$ ranibizumab. Patients were given a loading dose of 6 monthly injections and were then shifted to a PRN protocol. The study showed that after 12 months of follow-up there was a significant improvement in the VA in the ranibizumab-treated groups with a mean increase of 13.9 letters in both the $0.3 \mathrm{mg}$ and the $0.5 \mathrm{mg}$ dose compared with a mean of 7.3 letters in the sham $/ 0.5 \mathrm{mg}$ group. The visual gains achieved after the first 6 monthly doses could be maintained during the next 6 months using a pro re nata (PRN) protocol. The study also showed that delaying treatment affected patients adversely with the sham patients showing fewer gains compared with the other two groups. However, it is worth noting that these patients were being injected using a PRN protocol and that the sham $/ 0.5 \mathrm{mg}$ group received a mean of 3.7 injections during the observation period.

With regards to the adequate dose of ranibizumab, the study showed that there was also no difference between the 0.3-mg and the 0.5-mg doses. Also, in a recent study (RELATE) a cohort of chronic CRVO patients were injected with either a 0.5 -mg dose and a 2.0-mg dose of ranibizumab. ${ }^{12}$ The study showed that there was no significant difference between both groups with regards to visual outcome, although the 2.0-mg dose showed significant improvement with regards to central foveal thickness (CFT). Whether such an effect can be seen in patients with acute CRVO has yet to be seen. It would seem that patients with CRVO respond equally well to the $0.3-\mathrm{mg}, 0.5-\mathrm{mg}$, and the 2.0-mg doses of ranibizumab and that, at least for cases of acute CRVO, there is a limited role for increasing the dose of the injected drug.

In the CRUISE study it was noted that there was a slight drop in VA after patients were shifted from a monthly dosing regimen to a PRN dosing regimen. ${ }^{11}$ The authors raised the question whether patients could have achieved higher visual gains had they continued on a monthly dosing regimen for the first 12 months. This question was answered partly in the SHORE study that showed that after achieving visual and optical coherence tomography (OCT) stability criteria, both the PRN and monthly dosing regimens achieved similar results. However, the study protocol required all patients to be injected with at least 7 monthly loading doses, meaning that most patients were probably overtreated before randomization.

Furthermore, $15 \%$ of patients never met stability criteria for randomization and received monthly injections for the duration of the study. It is unclear whether these results could be replicated after a loading dose of 3 monthly injections. With no major RCT comparing 6 monthly loading doses with 3 monthly loading doses, there is no evidence to advocate using the latter.

One of the main problems with CRUISE was the highly selective cohort, which included non-ischemic CRVO patients and the exclusion of patients with afferent pupillary defects. In addition, the duration of CRVO in this study was $<3$ months, which makes it difficult to generalize these findings to patients with more chronic RVO and patients with ischemic RVO who may not respond to the same degree to anti-VEGF injections.

An extension study for CRUISE was the HORIZON study which included $87 \%$ of patients who participated in the CRUISE trial. ${ }^{13}$ Follow-ups were set every 3 months and patients were injected if CFT was $<250 \mu \mathrm{m}$ or there were signs of vision-threatening edema. Mean VA dropped from 16.2 letters and 14.9 letters in the $0.3 \mathrm{mg}$ and the $0.5 \mathrm{mg}$ doses to 12 letters and 8.2 letters, respectively. The mean change in BCVA letter score at 12 months from HORIZON baseline was $-4.2,-5.2$, and -4.1 in the sham/0.5-mg, 0.3/0.5-mg, and 0.5-mg treatment groups, respectively. At the end of the study VA gains in the sham $/ 0.5 \mathrm{mg}$ group were very similar to the $0.3 \mathrm{mg} / 0.5 \mathrm{mg}$ group; however, this likely occurred 
Table 1 Summary of the major Ranibizumab central retinal vein occlusion studies

\begin{tabular}{|c|c|c|c|}
\hline & Cruise $^{11}$ & Horizon $^{21}$ & Retain $^{14}$ \\
\hline Objective & $\begin{array}{l}\text { Assess the } 12 \text {-month efficacy and safety of } \\
\text { intraocular injections of } 0.3 \text { or } 0.5 \mathrm{mg} \\
\text { Ranibizumab in patients with macular edema } \\
\text { after central retinal vein occlusion (CRVO) }\end{array}$ & $\begin{array}{l}\text { To assess long-term safety and efficacy of } \\
\text { intraocular Ranibizumab injections in patients } \\
\text { with macular edema after retinal vein } \\
\text { occlusion (RVO) }\end{array}$ & $\begin{array}{l}\text { To determine long-term outcomes of patients with } \\
\text { Ranibizumab-treated retinal vein occlusion (RVO) }\end{array}$ \\
\hline Duration & 12 Months & 12 Months & 24 Months \\
\hline Intervention & $\begin{array}{l}\text { - Ranibizumab } 0.3 \mathrm{mg}(n=132) \text { Every } \\
4 \text { weeks for } 6 \text { months then pro re nata } \\
\text { (PRN) for another } 6 \text { months } \\
\text { - Ranibizumab } 0.5 \mathrm{mg}(n=130) \text { Every } \\
4 \text { weeks for } 6 \text { then PRN for another } \\
6 \text { months } \\
\text { - Sham }(n=130) \text { Every } 4 \text { weeks for } 6 \text { months } \\
\text { then PRN } 0.5 \mathrm{mg} \text { Ranibizumab for } \\
6 \text { months }\end{array}$ & $\begin{array}{l}304 \text { patients who completed CRUISE ( } 87 \%) \\
\text { were treated with } 0.5 \mathrm{mg} \text { Ranibizumab PRN. } \\
\text { Follow up every } 3 \text { months }\end{array}$ & $\begin{array}{l}32 \text { patients who completed HORIZON were injected } \\
\text { with } 0.5 \mathrm{mg} \text { Ranibizumab PRN } \\
\text { Follow up was monthly for the first year and then } \\
\text { every } 3 \text { months during the second year }\end{array}$ \\
\hline $\begin{array}{l}\text { Primary } \\
\text { end point }\end{array}$ & $\begin{array}{l}\text { Mean change from baseline } \\
\text { BCVA letter score at month } 6\end{array}$ & $\begin{array}{l}\text { Incidence and severity of ocular and } \\
\text { non-ocular AEs during the two year } \\
\text { extension period }\end{array}$ & $\begin{array}{l}\text { Mean improvement in best-corrected visual acuity } \\
\text { (BCVA) and percentage of patients with edema } \\
\text { resolution }\end{array}$ \\
\hline $\begin{array}{l}\text { Key inclusion } \\
\text { criteria }\end{array}$ & $\begin{array}{l}\text { - Age } \geq 18 \text { years } \\
\text { - Foveal center-involved Macular edema } \\
\text { secondary to central RVO. diagnosed } \\
\text { within } 12 \text { months prior to screening } \\
\text { - BCVA } 6 / 12 \text { to } 6 / 95 \text { Snellen equivalent } \\
\text { - Mean CRT } \geq 250 \mu \mathrm{m} \text { at screening and } \\
\text { Day } 0\end{array}$ & $\begin{array}{l}\text { - } 304 \text { patients who completed the } 12 \text {-month } \\
\text { CRUISE study } \\
\text { - Expectation by the investigator that the } \\
\text { patient could potentially benefit from } \\
\text { intravitreal anti-VEGF treatment }\end{array}$ & $\begin{array}{l}\text { - Patients who completed the Genentech-sponsored } \\
\text { Ranibizumab RVO trials. } 32 \text { patients with CRVO. }\end{array}$ \\
\hline Results & $\begin{array}{l}\text { Mean change from baseline BCVA letter } \\
\text { score at month } 12 \text { was } 13.9 \\
(11.2-16.5) \text { and } 13.9(11.5-16.4) \text { in the } 0.3 \mathrm{mg} \\
\text { and } 0.5 \mathrm{mg} \text { groups, respectively, and } 7.3 \\
(4.5-10.0) \text { in the sham } / 0.5 \mathrm{mg} \text { group } \\
(P<0.001 \text { for each Ranibizumab group } v \text { s } \\
\text { sham } / 0.5 \mathrm{mg})\end{array}$ & $\begin{array}{l}\text { In patients who completed month } 12 \text {, } \\
\text { the mean number of injections in the } \\
\text { sham } / 0.5-, 0.3 / 0.5-\text {, and } 0.5-\mathrm{mg} \text { groups was } \\
2.0,2.4 \text {, and } 2.1 \text { (branch RVO) and } 2.9,3.8 \text {, } \\
\text { and } 3.5 \text { (central RVO), respectively } \\
\text { The incidence of study eye ocular serious AEs } \\
\text { (SAEs) and SAEs potentially related to } \\
\text { systemic vascular endothelial growth factor } \\
\text { inhibition across treatment arms was } 2 \% \text { to } 9 \% \\
\text { and } 1 \% \text { to } 6 \% \text {, respectively }\end{array}$ & $\begin{array}{l}\text { With a mean follow-up of } 49.7 \text { months, } \\
14 \text { of } 32 \text { CRVO patients ( } 44 \% \text { ) had edema resolution, } \\
\text { with } 71 \% \text { receiving their last injection within } 2 \text { years } \\
\text { of treatment initiation. The mean number of injections } \\
\text { in unresolved patients in year } 4 \text { was } 5.9 \\
\text { Compared with patients with unresolved CRVO, } \\
\text { patients with resolved disease had greater } \\
\text { improvement in BCVA ( } 25.2 \text { vs } 4.3 \text { letters; } P=0.002) \text {, } \\
\text { and a greater percentage had a final BCVA of } 6 / 12 \text { or } \\
\text { better }(64.3 \% \text { vs } 27.8 \% ; P=0.04)\end{array}$ \\
\hline $\begin{array}{l}\% \text { of patients } \\
\text { gained } \\
\geq 15 \text { letters }\end{array}$ & $\begin{array}{l}\text { The percentage of patients who gained } \geq 15 \\
\text { letters from baseline BCVA at month } 12 \mathrm{was} \\
47.0 \% \text { and } 50.8 \% \text { in the } 0.3 \mathrm{mg} \text { and } 0.5 \mathrm{mg} \\
\text { groups }\end{array}$ & $\begin{array}{l}\text { In CRVO patients, at month } 12 \text { of HORIZON, } \\
\text { the percentage of patients who had an } \\
\text { improvement of } \geq 15 \text { letters from CRUISE } \\
\text { baseline was } 38.3 \% \text { (sham } / 0.5 \mathrm{mg}), 38.6 \% \\
(0.3 / 0.5 \mathrm{mg}) \text {, and } 45.1 \%(0.5 \mathrm{mg})\end{array}$ & $\begin{array}{l}\text { At the final visit, with a mean follow-up of } \\
51.4 \text { months, } 53.1 \% \text { of the } 32 \text { CRVO patients enrolled } \\
\text { in the RETAIN study gained } 15 \text { letters or more }\end{array}$ \\
\hline
\end{tabular}

Abbreviations: BCVA, best-corrected visual acuity; BRVO, branch retinal vein occlusion; CRVO, central retinal vein occlusion; ETDRS, Early Treatment Diabetic Retinopathy Study; FU, follow-up; N, number; RCT, randomised controlled trial.

because the $0.3 / 0.5 \mathrm{mg}$ group had more loss in visual acuity compared to the sham $/ 0.5 \mathrm{mg}$ group over that period. Patients with CRVO had a mean number of 2.9, 3.8 , and 3.5 injections in the sham $/ 0.5 \mathrm{mg}$, the $0.3 / 0.5 \mathrm{mg}$, and $0.5 \mathrm{mg}$ groups, respectively. The authors concluded that the loss in visual acuity was due to the longer 3-month follow-up period and the low number of injections. Perhaps a more suitable strategy would be monthly follow-ups until patients reach stability and then extending follow-up periods gradually to ensure adequate treatment.

The RETAIN study was an extension trial for HORIZON. ${ }^{14}$ There were 32 patients who completed the HORIZON trial and were enrolled in the RETAIN study, out of which only 27 completed 2 years of follow-up, for a total of 4 years since the start of CRUISE. The patients gained an overall increase of 14 letters that was not statistically significant from the gains achieved at the end of CRUISE, suggesting most patients were able to maintain their gains throughout HORIZON and RETAIN. Approximately $53.1 \%$ of patients gained 15 letters and $43.8 \%$ had a final BCVA of $6 / 12$ or better. RETAIN also stratified patients into those that had resolved edema for at least 6 months before the end of the study (which were considered as good responders) and those where the edema remained unresolved (which were considered as poor responders). Also, $43.8 \%$ of 32 patients had resolved edema of which $57 \%$ had their last injection in the first year, $14 \%$ had their last injection in the second year, and $21 \%$ in the third year. Patients with resolved edema showed rapid increase in visual acuity during the first 6 months that were maintained throughout the study. In the unresolved group (56\%), patients achieved much lower visual gains and required a mean number of 5.9 injections in year 4 to maintain them. This group can be categorized as non-responders or partial responders. 
Only 32 patients were enrolled into the RETAIN study and this represented about $10.5 \%$ of patients who were enrolled into HORIZON. This is a small fraction and may not be representative for all CRVO patients. In addition the cohort was mixed, patients from the sham $/ 0.5 \mathrm{mg}$, $0.3 / 0.5 \mathrm{mg}$ as well as the $0.5-\mathrm{mg}$ group were included and there was no stratification of the underlying subgroups. This combined with attrition bias and selection bias means that any conclusions made should be considered thoughtfully and further RCTs should be performed to confirm these findings. In addition, this is an extension study for CRUISE that only originally included non-ischemic CRVO patients. Although an estimated 1/3 of non-ischemic cases tend to convert to ischemic within 3 years, the studies (CRUISE, HORIZON, and RETAIN) do not report the number of nonischemic patients who converted to ischemic CRVO during the course of the study. ${ }^{15}$

The data from RETAIN show that poor responders $(\sim 50 \%)$ tend to require frequent injections and usually achieve low visual gains. It might be prudent to consider early change in therapeutics to ensure faster resolution of edema and better long-term outcomes. This would require detection of these patients as early as possible during the treatment regimen. Bhisitkul et al, ${ }_{1}^{16}$ reviewed the data from CRUISE to see whether the OCT at baseline or month 3 provides information that can predict visual outcomes. It was found that at month 3, OCT images provide predictive information for patients with CRVO in the 0.5-mg group but not the $0.3-\mathrm{mg}$ group. Approximately $78.5 \%(0.5 \mathrm{mg})$ and $71.2 \%(0.3 \mathrm{mg})$ of the CRVO patients in CRUISE had CFT $<250 \mu \mathrm{m}$ at 3 months and were referred to as early responders. The remaining patients who had residual fluid at 3 months were referred to as late or incomplete responders. Early responders had excellent long term visual outcomes regardless the dose of ranibizumab used whereas the late/incomplete responders did not fare as well especially when being treated with $0.3 \mathrm{mg}$ ranibizumab. Patients treated with $0.5 \mathrm{mg}$ ranibizumab with residual cystoid macular edema at 3 months achieved less visual gains at 6 months compared to the group without edema (13.1 letters vs 18.6 letters; $P=0.027$ ). A possible conclusion is that a 3-month OCT can give an indication as to whether the same treatment regimen should be continued or whether the patient should be shifted to an alternate treatment modality. On the basis of RETAIN, the same group of late/non-responders will require continuous treatment for years with modest visual gains and that perhaps an early change in treatment protocols might achieve better visual results.

\section{Aflibercept}

Two major RCTs evaluated the effects of aflibercept on CRVO namely COPERNICUS and GALILEO. ${ }^{17,18}$
A summary of the major aflibercept trials as well as a comparison with CRUISE, SCORE, and GENEVA is summarized in Table 2. It can be difficult to compare data between different trials, especially if baseline criteria and primary/secondary end points are different. The major Aflibercept trials both had a higher percentage of patients with ischemic CRVO; $15.5 \%$ in the COPERNICUS trial and $8.2 \%$ in the GALILEO trial compared with $0.5 \%$ in the CRUISE study. They also included patients with afferent pupillary defects that were excluded from CRUISE. The duration of edema was $<9$ months in both trials compared with $<3$ months in CRUISE. At 6 months the number of patients with $>15$ letters gain was $56.1 \%$ and $60.2 \%$ in the COPERNICUS and GALILEO trial respectively compared with $46.2 \%$ in CRUISE.

In the COPERNICUS study VA dropped as patients were shifted from monthly dosing to PRN dosing at 24 weeks in the $2 q 4$ aflibercept group (17.3 letter at week 24 and 16.2 letters at 52 weeks). ${ }^{17}$ Visual acuity further dropped at week 100 to reach 13 letters. In addition the number of patients with no fluid on OCT decreased from 74.5 to $34.3 \%$. These results were also mirrored by the GALILEO study that showed 18-letter improvements by 24 weeks that dropped to 16.9 letters at the end of the first year and 13.7 letters at 76 weeks. ${ }^{18}$ In addition, $80 \%$ of patients had no fluid on OCT at week 24 compared with $60.2 \%$ at 76 weeks. This deterioration in visual acuity in both studies can be explained by the extended duration of follow-ups in studies. After the first year patients in COPERNICUS were followed every 3 months and patients in GALILEO were followed every 2, suggesting a quarterly protocol is not a reasonable strategy to maintain these visual gains. A modified goal would be to identify patients that need more frequent follow-ups and determine a treatment interval that would prevent fluid recurrences while maintaining gains. This strategy is akin to the observe and plan strategy (OAP) previously proposed for age-related macular degeneration; a modification of the treat and extend protocol, that follows up patients to determine the time point of recurrences and fixes an injection 2 weeks prior. ${ }^{19}$ Although this strategy would seem applicable to all patients, it will likely be more effective in patients who develop multiple recurrences.

Although there is insufficient data to say that aflibercept is better than ranibizumab, it is at least equally effective and at most the only anti-VEGF tested in ischemic CRVO patients in RCTs. Subgroup analyses investigating the impact of retinal perfusion status on vision in COPERNICUS and GALILEO showed that both groups experienced similar gains in visual acuity and anatomical resolution of macular edema with anti-VEGF therapy. ${ }^{17,18}$ 


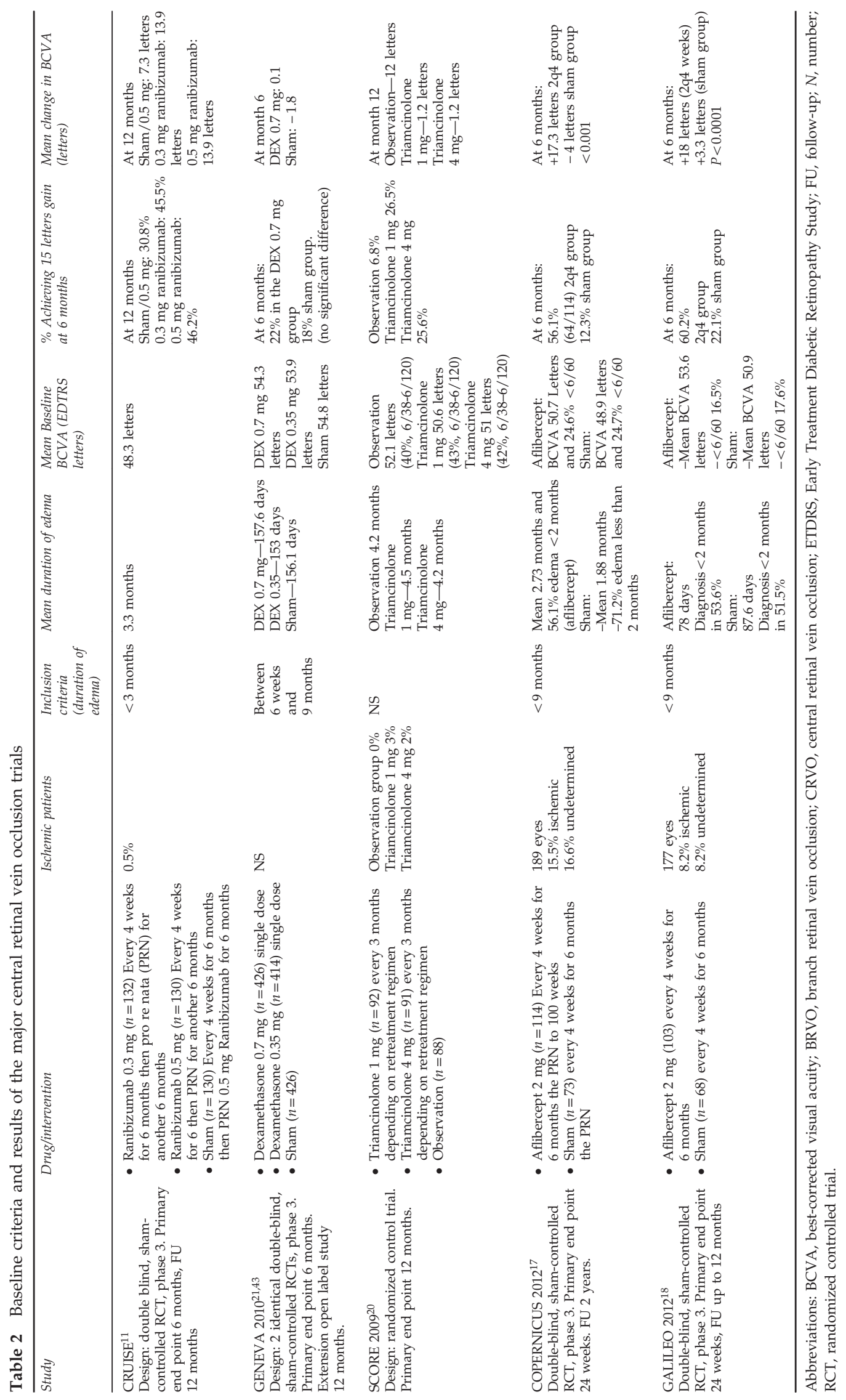




\section{Steroids}

The efficacy of steroids has been demonstrated previously in two major trials; SCORE and GENEVA. ${ }^{20,21}$ The SCORE study looked at the effects of two doses of triamcinolone (1 and $4 \mathrm{mg}$ ) compared with observation and the results showed that at 1 year 27 and $26 \%$ of subjects had a 15-letter improvement compared with the observation group that showed $7 \% .{ }^{20}$ In addition, the mean visual gain was -1.2 letters in both the 1 and $4 \mathrm{mg}$ groups compared with -7 letters in the observation group. There was no difference in OCT macular thickness between the different groups at 1 year, which reinforces a moderate correlation between OCT thickness and visual acuity in patients with retinal vein occlusion. ${ }^{22}$

In the GENEVA trial dexamethasone implants (Ozurdex, Allergan, Inc., Irvine, CA, USA) were used but included both BRVO patients and CRVO patients (34\%) in their results. ${ }^{21}$ The combined results showed that at 6 months there was a 15-letter improvement in $41 \%$ and $40 \%$ of patients using the 0.7 and $0.35 \mathrm{mg}$ groups, respectively, compared with $23 \%$ in the sham group. A subgroup analysis showed that for CRVO patients $29 \%$ in the $0.7 \mathrm{mg}$ and $33 \%$ in the $0.35 \mathrm{mg}$ groups had a 15-letter improvement compared with $9 \%$ the sham group at 2 months. However, at 6 months these differences were not significant with percentages dropping to $18 \%, 17 \%$, and $12 \%$ for the $0.7 \mathrm{mg}, 0.35 \mathrm{mg}$ and the sham group, respectively. A post hoc analysis showed that patients with more recent onset edema responded better than patients with more chronic edema. ${ }^{23}$ These results were also mirrored by the SCORE study indicating that steroids might be more effective in recent onset edema. ${ }^{20,21}$

No large randomized studies have compared between steroids and anti-VEGFs. A small study by Gado AS and Macky TA was conducted on 60 patients with nonischemic CRVO in which patients were randomized into two groups, one receiving Bevazicumab and the other receiving Ozurdex implants. The study showed that there was no significant difference in BCVA or macular thickness between the two groups at 6 months. However, there was a significantly higher IOP in the dexamethasone group. Another study by Ding et al, ${ }^{24}$ conducted on 32 patients showed similar results at 9 months. A third study by Chiquet et al, conducted on 102 patients randomized to receive anti-VEGFs and Dexamethasone implants showed that at 3 months there was significantly better visual outcomes in the DEX group with no difference in the CFT. These differences were not maintained and by the first year there was no difference in anatomical or visual outcomes. However, an elevated IOP was more frequent in the DEX group (21\%) compared with the anti-VEGF group $(3 \%, P=0.008) .{ }^{25}$
With regards to the major RCTs, the outcomes between both drugs in the CRUISE and SCORE studies are different; whether this is because of different baseline criteria and patient characteristics or because of different drug efficacies has yet to be demonstrated. While the mean VA gain in the SCORE study for the 1- and 4-mg dose was -1.2 , the mean VA gain in CRUISE was +13.9 letters in the $0.3 \mathrm{mg}$ and the $0.5 \mathrm{mg}$ Ranibizumab groups. Percentage of patients achieving $>15$ letter gains was 47 and $51 \%$ in the $0.3 \mathrm{mg}$ and the $0.5 \mathrm{mg}$ ranibizumab groups in CRUISE compared with 26 and $27 \%$ in the 1 and $4 \mathrm{mg}$ triamcinolone groups. ${ }^{11,20}$ In addition, 20 and $35 \%$ in the 1 and $4 \mathrm{mg}$ triamcinolone groups required initiation of IOP lowering medications compared with $8 \%$ in the observation group. A recent study by Thom et al, ${ }^{26}$ attempted to compare between trials using a combination of multinomial and indirect Bayesian comparison models. It showed that there was a trend for greater ranibizumab associated visual gains compared with dexamethasone at months 1 and 6 in a common clinical context, although results were not classically significant. Thus, even if the visual gains were similar, steroids would still not be the preferred first line choice for many because of the higher intraocular complication rate.

\section{Alternate treatment regimens}

Response can be variable amongst patients and defining this response is crucial to tailoring a patient-centric treatment regimen. A simple definition could be the one described earlier that categorizes patients with a CFT $>250 \mu \mathrm{m}$ after three injections as late or partial/ non-responders and those with a CFT of $<250 \mu \mathrm{m}$ as early or good responders. ${ }^{16}$ As previously mentioned patients showing fluid at 3 months fared worse at 6 months and 12 months with less visual gains and required more frequent injections. To further stratify this group of poorly responsive patients we suggest the definition used by Sharareh et al that suggested that patients who have $<1 \%$ reduction in $\mathrm{MO}$ after injections are to be deemed non-responders and patients who respond with at least a $10 \%$ reduction in $\mathrm{MO}$ to be categorized as partial responders. ${ }^{27}$ With that concept in mind we will explore some of the alternate treatment modalities that can be used in patient deemed nonresponsive or partially responsive after three loading doses of anti-VEGFs.

\section{Laser therapy}

There are two distinct laser strategies: GRID and scatter PRP. GRID laser aims to reduce macular edema and improve VA, and scatter PRP aims to reduce VEGF load by ablating peripheral ischemic retina. 
The first major study to assess the effects of GRID photocoagulation in improving visual acuity in eyes with macular edema was the CVOS study. ${ }^{15}$ The study consisted of 155 eyes divided into two groups; a laser GRID group and an observation group. At the conclusion of the study there were no differences between the treated and untreated eyes in terms of visual acuity. However, treatment did reduce angiographic evidence of macular edema, although this did not translate to improvements in visual outcomes. Subgroup analysis showed that the visual acuity outcomes were consistent over all subgroups (which included duration of CRVO and degree of peripheral ischemia) except age. However, this study used laser as a monotherapy and with very high CFTs the effectiveness of laser therapy might have been reduced. It would seem reasonable to explore laser therapy after the CFT has been reduced by anti-VEGF therapy. A pilot study looking at long-term effects of early intervention with intravitreal bevazicumab followed by grid and pan-retinal photocoagulation (PRP) in nine eyes with non-ischemic CRVO showed excellent improvement in VA and anatomy. ${ }^{28}$ Although the results should be taken with caution, perhaps a more long-term RCT could show how effective this strategy can be.

With regards to scatter PRP, the RELATE study showed that there was no added benefit with regard to bestcorrected visual acuity (BCVA), resolution of edema, or number of injections in the ranibizumab plus laser group compared to the ranibizumab only group. ${ }^{12}$ Scatter PRP exacerbated the macular edema and did not decrease it as would be expected. Another small prospective study by Spaide showed similar findings. ${ }^{29}$ Although the cohort in the RELATE study was mainly chronic CRVO patients (mean of 15 months duration), the authors suggested that because of the exacerbation of edema post laser that conducting a trial on acute patients may not be warranted. ${ }^{12}$

\section{Switching to steroids}

The difference in visual outcomes as well as the higher incidence of complications with steroids suggests that anti-VEGF would be the more preferable first line drug. Steroids can be used as a second line drug in resistant cases or as an adjunct from the start. A retrospective study by Sharareh et al, ${ }^{27}$ looked at 18 patients categorized as complete or partial responders to Bevazicumab that were given Dexamethasone implants. The study showed that both subgroups responded with an improvement in both central macular thickness (average $147 \mu \mathrm{m}$ ) and visual acuity (mean improvement of $0.25 \log$ MAR). The OMAR study compared between the effects of Ozurdex and triamcinolone acetonide in cases of refractory cystoid macular edema despite repeated bevazicumab therapy due to retinal vein occlusion. ${ }^{30}$ It showed that adding steroids improved central macular thickness significantly $(P<0.0001)$ although final BCVA did not change significantly after steroid introduction $(P=0.06)$. There was no difference between triamcinolone (TA) and dexamethasone (DEX) regarding anatomic or functional outcomes.

As an adjunct, a case series by Singer et al, ${ }^{31}$ showed that dexamethasone implant with bevazicumab showed a synergistic effect in CRVO and BRVO patients, increasing VA and prolonging the time between injections, compared with either of these medications alone. In addition, $\sim 55 \%$ of patients had a maximum visual acuity gain of 3 letters with a mean increase of 3.4 lines, and $18 \%$ of patients did not require re-injection. Another study by Maturi et al, ${ }^{32}$ compared between patients who received bevazicumab alone and patients who received combination therapy with dexamethasone implants; at 6 months there was a greater reduction in mean CMT in the combined group compared with the monotherapy group, despite no significant differences in VA.

These data show that dexamethasone implants and to a lesser extent TA can be a suitable option in resistant cases either as monotherapy or an adjunct to bevazicumab or ranibizumab.

\section{Switching between anti-VEGFs}

Switching between anti-VEGFs as a strategy has been used in diabetic macular edema (DMO) and in age-related macular degeneration (AMD) with variable results. ${ }^{33-37}$ There have been no large-scale studies exploring the effects of switching in CRVO. A single case series looked at the benefit of switching to ranibizumab in cases resistant to bevacizumab and showed that there was an improvement in both visual acuity and anatomy in these patients. ${ }^{38}$ Two case series each with six cases have looked into the effects of switching to aflibercept in ranibizumab resistant cases. One study showed marked improvement in edema with modest improvement in visual acuity and the other which investigated a cohort of ischemic CRVO patients showed marked improvements in both edema and VA. ${ }^{39,40}$ Although the numbers were small, the initial data suggest switching cases to Aflibercept may be a treatment option in non-responsive cases especially if they were ischemic.

A larger study published by Papakostas et al, included 42 patients with CRVO non-responsive to bevacizumab/ ranibizumab that were switched to aflibercept. ${ }^{41}$

The median number of injections prior to the switch was seven and the group included $70 \%$ that were partially responsive and $9.5 \%$ that were non-responsive to bevacizumab and/or ranibizumab. The remaining were initially responsive but on recurrence failed to 
show a response to either drug. Post switch visual acuity improved at 1 month but was no longer statistically significant at the end of follow-up. However, anatomically there was a significant and sustained improvement from median CRT 536 to $279 \mu \mathrm{m}$ at the end of follow-up $(P=0.0013)$. Interestingly, the median interval between injections increased from 5.6 weeks to 7.6 weeks $(P<0.0001)$. Similarly Pfau et al, ${ }^{42}$ switched 13 patients partially responding to ranibizumab/ bevacizumab to aflibercept and attempted to increase the duration of treatment using a treat and extend protocol. Patients showed statistically significant decrease in anatomy and showed an improvement in visual acuity by +10.38 letters $(P=0.021)$. In addition, the duration between injections increased by 0.51 months and the relapse-free interval increased by 3.02 weeks $(P=0.003)$.

Therefore it would seem that most studies demonstrated the anatomical efficacy and in some the visual benefits of switching to aflibercept. However, these studies were all retrospective and switching was performed after a prolonged period of initial treatment. This highlights the importance of conducting a prospective study that aims at early switching to study its efficacy in the non/partial-response group. Furthermore, it highlights that in anti-VEGF dependent cases aflibercept is an interesting choice because it allows for less frequent injections. However, the benefit of achieving

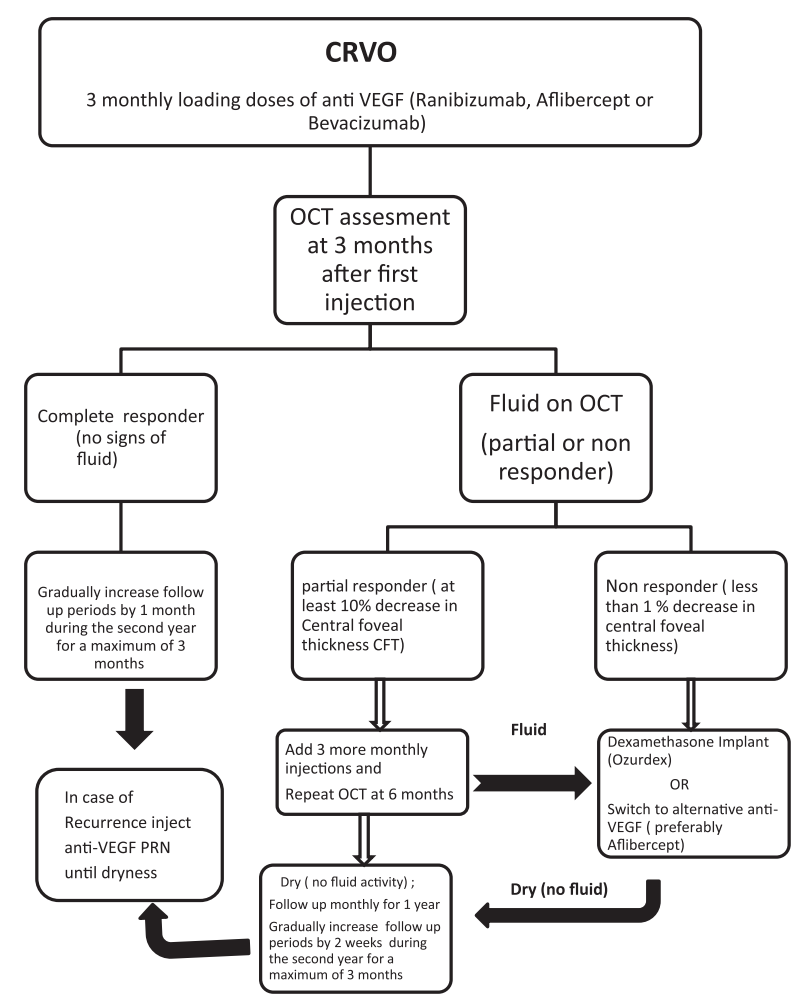

Figure 1 Proposed algorithm for treating central retinal vein occlusion. anatomical improvement without the concurrent visual improvement means that perhaps the treatment goals for some patients would be maintenance of vision as opposed to improving it.

\section{Summary}

On the basis of the current review of previous trials we devised a treatment algorithm that summarizes all the findings from the major trials as demonstrated in Figure 1. Our recommended protocol for CRVO patients would include a loading dose of three monthly injections of ranibizumab or aflibercept or unlicensed bevazicumab. Ranibizumab and aflibercept are FDA and EMA approved as well as being recommended by NICE (NICE TA and NICE TA 305) for the treatment of visual impairment due to MO secondary to CRVO. Bevacizumab is unlicensed for intravitreal injections and is considered as a first line drug if ranibizumab or aflibercept are unavailable or due to economic considerations. At 3 months patients should be assessed for fluid on OCT. We believe that there are two main patterns of response; good or early responders and partial/non-responders based on the data from Bhisitkul et al that classified patients based on CFT after 3 monthly injections. ${ }^{16}$ The group with fluid at 3 months will be further categorized using the definition proposed by Sharareh et al into those who have less than a $1 \%$ reduction in $\mathrm{MO}$ after 3 injections (the non-responders) and those who respond with at least a $10 \%$ reduction in MO (the partial responders). ${ }^{27}$ Patients showing no fluid activity (early responders) after 3 monthly injections should be followed up monthly and injected using a PRN protocol for the first year and with gradual monthly extensions of the follow-up period during the second year to maintain gains. Patients showing residual fluid at 3 months should be categorized into partial or non-responders. Partial responders may complete the 6 loading doses as previously used in the major studies like CRUISE, after which they should be reassessed. ${ }^{11}$ If they still show fluid activity, then they may be offered a switch in treatment protocols. Non-responders after 3 monthly injections may be offered one of three choices; dexamethasone implants alone or in combination with bevazicumab/ranibizumab or a switch to an alternate anti-VEGF (preferably aflibercept if ranibizumab or unlicensed bevacizumab was used). Patients in the non-responders and partial responders group should be followed monthly for 1 year at least after achieving dryness. These patients should have their follow-up periods extended very cautiously with an attempt to identify a fixed period of recurrence before which patients will receive an anti-VEGF injection. The choice will 
depend on the agent that achieved dryness for that particular patient.

We also recommend more randomized control studies to look into the effect of perfusion status as well as the duration of edema on the response of patients to various treatment modalities. Exploring options such as switching to Aflibercept or adjunct dexamethasone would offer more options for better visual acuity outcomes. More prospective studies are needed that look into the effects of early switching as well as a comparison between switching to an alternate anti-VEGF agent or Ozurdex as this would help us better understand our treatment options. On the basis of the data we do not recommend scatter PRP or Grid laser until more RCTs can show a benefit in terms of visual outcomes or reducing treatment load.

Finally, we advocate a patient-centric individualized approach in dealing with CRVO and awareness that this is a chronic disease that requires close follow-up and continuous care for years to ensure that early successes are not lost down the line.

\section{Conflict of interest}

The authors declare no conflict of interest.

\section{Acknowledgements}

Dr Singh serves as a consultant for Regeneron, Genentech, Alcon, Shire, and Thrombogenics. He receives research support from Regeneron, Genentech, and Alcon.

\section{References}

1 Hayreh SS. Prevalent misconceptions about acute retinal vascular occlusive disorders. Prog Retin Eye Res 2005; 24(4): 493-519.

2 Rogers S, McIntosh RL, Cheung N, Lim L, Wang JJ, Mitchell $\mathrm{P}$ et al. The prevalence of retinal vein occlusion: pooled data from population studies from the United States, Europe, Asia, and Australia. Ophthalmology 2010; 117(2): 313-319 e311.

3 Campochiaro PA, Hafiz G, Shah SM, Nguyen QD, Ying H, Do DV et al. Ranibizumab for macular edema due to retinal vein occlusions: implication of VEGF as a critical stimulator. Mol Ther 2008; 16(4): 791-799.

4 Hayreh SS, Zimmerman MB, Podhajsky P. Incidence of various types of retinal vein occlusion and their recurrence and demographic characteristics. Am J Ophthalmol 1994; 117(4): 429-441.

5 Baseline and early natural history report. The Central Vein Occlusion Study. Arch Ophthalmol 1993; 111(8): 1087-1095.

6 Hayreh SS, Podhajsky PA, Zimmerman MB. Natural history of visual outcome in central retinal vein occlusion. Ophthalmology 2011; 118(1): 119-133 e111-112.

7 Natural history and clinical management of central retinal vein occlusion. The Central Vein Occlusion Study Group. Arch Ophthalmol 1997; 115(4): 486-491.
8 Ferrara N, Damico L, Shams N, Lowman H, Kim R. Development of ranibizumab, an anti-vascular endothelial growth factor antigen binding fragment, as therapy for neovascular age-related macular degeneration. Retina 2006; 26(8): 859-870.

9 Ferrara N, Hillan KJ, Gerber HP, Novotny W. Discovery and development of bevacizumab, an anti-VEGF antibody for treating cancer. Nat Rev Drug Discov 2004; 3(5): 391-400.

10 Holash J, Davis S, Papadopoulos N, Croll SD, Ho L, Russell M et al. VEGF-Trap: a VEGF blocker with potent antitumor effects. Proc Natl Acad Sci USA 2002; 99(17): 11393-11398.

11 Campochiaro PA, Brown DM, Awh CC, Lee SY, Gray S, Saroj $\mathrm{N}$ et al. Sustained benefits from ranibizumab for macular edema following central retinal vein occlusion: twelve-month outcomes of a phase III study. Ophthalmology 2011; 118(10): 2041-2049.

12 Campochiaro PA, Hafiz G, Mir TA, Scott AW, Solomon S, Zimmer-Galler I et al. Scatter photocoagulation does not reduce macular edema or treatment burden in patients with retinal vein occlusion: the RELATE trial. Ophthalmology 2015; 122(7): 1426-1437.

13 Heier JS, Campochiaro PA, Yau L, Li Z, Saroj N, Rubio RG et al. Ranibizumab for macular edema due to retinal vein occlusions: long-term follow-up in the HORIZON trial. Ophthalmology 2012; 119(4): 802-809.

14 Campochiaro PA, Sophie R, Pearlman J, Brown DM, Boyer DS, Heier JS et al. Long-term outcomes in patients with retinal vein occlusion treated with ranibizumab: the RETAIN study. Ophthalmology 2014; 121(1): 209-219.

15 Evaluation of grid pattern photocoagulation for macular edema in central vein occlusion. The Central Vein Occlusion Study Group M report. Ophthalmology 1995; 102(10): 1425-1433.

16 Bhisitkul RB, Campochiaro PA, Shapiro H, Rubio RG. Predictive value in retinal vein occlusions of early versus late or incomplete ranibizumab response defined by optical coherence tomography. Ophthalmology 2013; 120(5): 1057-1063.

17 Heier JS, Clark WL, Boyer DS, Brown DM, Vitti R, Berliner AJ et al. Intravitreal aflibercept injection for macular edema due to central retinal vein occlusion: two-year results from the COPERNICUS study. Ophthalmology 2014; 121(7): 1414-1420 e1411.

18 Korobelnik JF, Holz FG, Roider J, Ogura Y, Simader C, Schmidt-Erfurth $\mathrm{U}$ et al. Intravitreal aflibercept injection for macular edema resulting from central retinal vein occlusion: one-year results of the Phase 3 GALILEO Study. Ophthalmology 2014; 121(1): 202-208.

19 Mantel I, Niderprim SA, Gianniou C, Deli A, Ambresin A. Reducing the clinical burden of ranibizumab treatment for neovascular age-related macular degeneration using an individually planned regimen. Br J Ophthalmol 2014; 98(9): 1192-1196.

20 Ip MS, Scott IU, VanVeldhuisen PC, Oden NL, Blodi BA, Fisher $\mathrm{M}$ et al. A randomized trial comparing the efficacy and safety of intravitreal triamcinolone with observation to treat vision loss associated with macular edema secondary to central retinal vein occlusion: the Standard Care vs Corticosteroid for Retinal Vein Occlusion (SCORE) study report 5. Arch Ophthalmol 2009; 127(9): 1101-1114.

21 Haller JA, Bandello F, Belfort Jr R, Blumenkranz MS, Gillies M, Heier J et al. Randomized, sham-controlled trial of dexamethasone intravitreal implant in patients with macular 
edema due to retinal vein occlusion. Ophthalmology 2010; 117(6): 1134-1146 e1133.

22 Scott IU, VanVeldhuisen PC, Oden NL, Ip MS, Blodi BA, Jumper JM et al. SCORE Study report 1: baseline associations between central retinal thickness and visual acuity in patients with retinal vein occlusion. Ophthalmology 2009; 116(3): 504-512.

23 Yeh WS, Haller JA, Lanzetta P, Kuppermann BD, Wong TY, Mitchell $\mathrm{P}$ et al. Effect of the duration of macular edema on clinical outcomes in retinal vein occlusion treated with dexamethasone intravitreal implant. Ophthalmology 2012; 119(6): 1190-1198.

24 Ding X, Li J, Hu X, Yu S, Pan J, Tang S. Prospective study of intravitreal triamcinolone acetonide versus bevacizumab for macular edema secondary to central retinal vein occlusion. Retina 2011; 31(5): 838-845.

25 Chiquet C, Dupuy C, Bron AM, Aptel F, Straub M, Isaico $\mathrm{R}$ et al. Intravitreal dexamethasone implant versus anti-VEGF injection for treatment-naive patients with retinal vein occlusion and macular edema: a 12-month follow-up study. Graefes Arch Clin Exp Ophthalmol 2015; 253: 2095-2102.

26 Thom HH, Capkun G, Nixon RM, Ferreira A. Indirect comparisons of ranibizumab and dexamethasone in macular oedema secondary to retinal vein occlusion. BMC Med Res Methodol 2014; 14: 140.

27 Sharareh B, Gallemore R, Taban M, Onishi S, Wallsh J. Recalcitrant macular edema after intravitreal bevacizumab is responsive to an intravitreal dexamethasone implant in retinal vein occlusion. Retina 2013; 33(6): 1227-1231.

28 Shah NJ, Shah UN. Long-term effect of early intervention with single intravitreal injection of bevacizumab followed by panretinal and macular grid photocoagulation in central retinal vein occlusion (CRVO) with macular edema: a pilot study. Eye (Lond) 2011; 25(2): 239-244.

29 Spaide RF. Prospective study of peripheral panretinal photocoagulation of areas of nonperfusion in central retinal vein occlusion. Retina 2013; 33(1): 56-62.

30 Ozkok A, Saleh OA, Sigford DK, Heroman JW, Schaal S. THE OMAR STUDY: Comparison of Ozurdex and Triamcinolone Acetonide for Refractory Cystoid Macular Edema in Retinal Vein Occlusion. Retina 2015; 35(7): 1393-1400.

31 Singer MA, Bell DJ, Woods P, Pollard J, Boord T, Herro A et al. Effect of combination therapy with bevacizumab and dexamethasone intravitreal implant in patients with retinal vein occlusion. Retina 2012; 32(7): 1289-1294.

32 Maturi RK, Chen V, Raghinaru D, Bleau L, Stewart MW. A 6-month, subject-masked, randomized controlled study to assess efficacy of dexamethasone as an adjunct to bevacizumab compared with bevacizumab alone in the treatment of patients with macular edema due to central or branch retinal vein occlusion. Clin Ophthalmol 2014; 8: 1057-1064.

33 Aslankurt M, Aslan L, Aksoy A, Erden B, Cekic O. The results of switching between 2 anti-VEGF drugs, bevacizumab and ranibizumab, in the treatment of neovascular age-related macular degeneration. Eur J Ophthalmol 2013; 23(4): 553-557.

34 Bakall B, Folk JC, Boldt HC, Sohn EH, Stone EM, Russell SR et al. Aflibercept therapy for exudative age-related macular degeneration resistant to bevacizumab and ranibizumab. Am J Ophthalmol 2013; 156(1): 15-22.e11.

35 Batioglu F, Demirel S, Ozmert E, Abdullayev A, Bilici S. Short-term outcomes of switching anti-VEGF agents in eyes with treatment-resistant wet AMD. BMC Ophthalmol 2015; 15: 40 .

36 Hanhart J, Chowers I. Evaluation of the response to ranibizumab therapy following bevacizumab treatment failure in eyes with diabetic macular edema. Case Rep Ophthalmol 2015; 6(1): 44-50.

37 Dhoot DS, Pieramici DJ, Nasir M, Castellarin AA, Couvillion S, See RF et al. Residual edema evaluation with ranibizumab $0.5 \mathrm{mg}$ and $2.0 \mathrm{mg}$ formulations for diabetic macular edema (REEF study). Eye (Lond) 2015; 29(4): 534-541.

38 De Niro JE, Fu AD, Johnson RN, McDonald HR, Jumper JM, Cunningham $\mathrm{E}$ et al. Intravitreous ranibizumab for persistent macular edema in retinal vein occlusion unresponsive to bevacizumab. Retin Cases Brief Rep 2013; 7(3): 220-224.

39 Eadie JA, Ip MS, Kulkarni AD. Response to aflibercept as secondary therapy in patients with persistent retinal edema due to central retinal vein occlusion initially treated with bevacizumab or ranibizumab. Retina 2014; 34(12): 2439-2443.

40 Lehmann-Clarke L, Dirani A, Mantel I, Ambresin A. The effect of switching ranibizumab to aflibercept in refractory cases of macular edema secondary to ischemic central vein occlusion. Klin Monbl Augenheilkd 2015; 232(4): 552-555.

41 Papakostas TD, Lim L, van Zyl T, Miller JB, Modjtahedi BS, Andreoli CM et al. Intravitreal aflibercept for macular oedema secondary to central retinal vein occlusion in patients with prior treatment with bevacizumab or ranibizumab. Eye (Lond) 2016; 30(1): 79-84.

42 Pfau M, Fassnacht-Riederle H, Becker MD, Graf N, Michels S. Clinical outcome after switching therapy from ranibizumab and/or bevacizumab to aflibercept in central retinal vein occlusion. Ophthalmic Res 2015; 54(3): 150-156.

43 Haller JA, Bandello F, Belfort Jr R, Blumenkranz MS, Gillies M, Heier J et al. Dexamethasone intravitreal implant in patients with macular edema related to branch or central retinal vein occlusion twelve-month study results. Ophthalmology 2011; 118(12): 2453-2460. 